\title{
EPIDEMIOLOGY OF INVASIVE PNEUMOCOCCAL DISEASE IN CZECH CHILDREN UNDER 5 YEARS OF AGE AFTER ROUTINE IMMUNISATION
}

\author{
Marek Petráš ${ }^{1}$, Věra Adámková2 \\ ${ }^{1}$ Second Faculty of Medicine, Charles University in Prague, Prague, Czech Republic \\ ${ }^{2}$ Institute for Clinical and Experimental Medicine, Prague, Czech Republic
}

\begin{abstract}
SUMMARY
Background: The introduction of the surveillance of invasive pneumococcal diseases (IPD) in 2007 has helped to monitor changes in serotype occurrence in the Czech population and assess the impact of routine immunisation against IPD on the child population under 5 years of age.

Methods: Observational study of childhood IPD in the Czech Republic based on the state public health surveillance data comparing preimmunisation (2007-2008) and post-immunisation (2012-2013) periods.

Results: In the child population there was an overall decline in IPD occurrence of $46.6 \%(95 \% \mathrm{Cl} 63.4-21.9)$ observed during the post-immunisation period. There was even greater decrease of $71.6 \%(95 \% \mathrm{Cl} 50.4-83.8)$ in vaccinated children. In the post-immunisation period, Prevenar 13 (PCV13) and Synflorix (PCV10) contributed to a vaccine-type IPD reduction of $95.4 \%(95 \% \mathrm{Cl} 67.0-99.4)$ and $76.9 \%(95 \% \mathrm{Cl} 36.0-91.7)$, respectively, compared to unimmunised children. The occurrence of 10 serotypes contained in both commercial vaccines also decreased in unvaccinated children by $61.4 \%$ (95\% $\mathrm{Cl} 14.5-82.6)$. However, a rise in the risk of non-vaccine and unspecified serotype acquisition by up to $153 \%$ was revealed in unimmunised children when comparing post-immunisation and pre-immunisation periods.

Conclusions: The findings suggest a shift in IPD caused either by vaccine or non-vaccine serotypes between immunised and unimmunised populations of children, which could result in increased incidence of IPD caused by non-vaccine serotypes. Therefore, routine immunisation using only one vaccine with broader serotype coverage together with a higher vaccination rate could raise hopes of further decrease in IPD in the child population.
\end{abstract}

Key words: invasive pneumococcal disease, pneumococcal conjugate vaccines, vaccine and non-vaccine serotypes

Address for correspondence: M. Petráš, Charles University in Prague, Second Faculty of Medicine, V Úvalu 84, 15006 Prague 5, Czech Republic. E-mail: petras@vakciny.net

http://dx.doi.org/10.21101/cejph.a4161

\section{INTRODUCTION}

Infant immunisation against invasive pneumococcal disease (IPD) with conjugate vaccines has been available in the Czech Republic since 2005. The pneumococcal conjugate vaccines (PCV) introduced into the childhood immunisation programme in 2010 have enabled vaccination costs to be mostly or completely covered by health insurance for all infants younger than one year. Vaccination in the Czech Republic, which had been initially realised with only one vaccine, Prevenar (PCV7), has been conducted with Synflorix (PCV10) since 2009 and Prevenar 13 (PCV13) since 2010.

The aim of this observational retrospective study is to assess the impact of routine immunisation on IPD incidence in children under 5 years of age and to verify if there have been any changes in vaccine and non-vaccine serotypes occurrence in the child population.

\section{MATERIALS AND METHODS}

\section{IPD Incidence}

The occurrence of invasive pneumococcal disease has been monitored within the National Surveillance Programme since 2007 , conducted as pilot monitoring in the first two years. Analyzed data of serotypes within the age group of 0-4 years were obtained from publications in the years 2009-2014 (1-6). Cases of IPD were stratified according to vaccine serotypes (VT) and non-vaccine serotypes (NVT). Overall, 11 IPD cases with no serotype specification were assigned to non-typed IPD cases (NT). Although the related publication did not report whether children with IPD recorded in 2007-2008 had been immunised, it might be assumed that in this pre-immunisation period the children were not vaccinated. The reason for this assumption arises from the low vaccination rate resulting from the absence of routine 
immunisation in these years. Cases reported in 2012 and 2013 were accompanied by information on whether the infant or child was immunised and the type of commercial vaccine administered (Table 1). Overall IPD occurrence in 2007-2008 reported for both years together totalled 70 cases, from which 46 and 58 cases were caused by 10 and 13 vaccine serotypes, respectively. Therefore, the analysis assessed the situation before and after routine immunisation over a two-year period, i.e. 2007-2008 (pre-immunisation period) and 2012-2013 (post-immunisation period), respectively.

\section{PCV Immunisation}

Routine pneumococcal vaccination has been in effect since the beginning of 2010. Infants born in 2009 were also considered eligible for immunisation. In the first year of routine immunisation, PCV7 and PCV10 vaccines were used. Replacement of PCV7 with the PCV13 vaccine began in the second year of immunisation and partial immunisation with PCV7 was completed with the PCV13 vaccine. Therefore, this assessment focused on the PCV10 and PCV13 immunisations.

Only four PCV7 vaccinated children acquired IPD, as reported in 2012-2013. These cases were caused by the non-vaccine serotype and therefore assigned to children who were not immunised with PCV10 or PCV13. In addition, two cases with unknown status of immunisation and no IPD serotype specification were excluded from the analysis.

The vaccination coverage among children under 5 years of age was considered to be equal for both vaccines and estimated as $100 \% \mathrm{x}$ (1-IPDpost/IPDpre), where IPDpre and IPDpost were the number of cases in unvaccinated children from the pre- and post-immunisation period, respectively.

\section{Statistical Analysis}

Vaccination impact was assessed with a crude or birth cohort adjusted odds ratio (OR or aOR), including 95\% confidence intervals (CIs). The number of living infants and children in the years 2002-2013 was obtained from the Czech Statistical Office database. The vaccination rate of each birth cohort was set as follows: $0 \%, 50 \%, 80 \%, 80 \%, 80 \%$ and $80 \%$ for birth cohorts in the years 2008, 2009, 2010, 2011, 2012 and 2013, respectively.
The decline of IPD was estimated as 1 minus the OR, expressed as a percentage. Analyses were performed using StatsDirect Statistical Software, version 3.0.117 (StatsDirect Ltd, UK).

\section{RESULTS}

When comparing pre- and post-immunisation periods, there was an IPD decline of $46.6 \%$ (95\% CI 21.9-63.4) in the general child population under 5 years of age. The odds ratio, determined independently of the vaccination rate, was $0.53(95 \% \mathrm{CI}$ $0.37-0.78$ ). A much higher decrease of $71.6 \%$ (95\% CI 50.4-83.7) was observed in the vaccinated children, i.e. the OR of $0.28(95 \%$ CI $0.16-0.50)$. The vaccination rate reached $68.6 \%(95 \% \mathrm{CI}$ 56.4-79.2) in the post-immunisation period.

Although a slightly higher overall reduction of $73.5 \%$ (95\% CI 42.4-87.8) in children vaccinated with PCV13 compared to those vaccinated with PCV10 was observed (Table 2), the adjusted odds ratio of 0.89 (95\% CI 0.34-2.30) between those immunised with both PCV vaccines did not exhibit any statistical difference from IPD cases between those in the years 2012-2013.

Moreover, PCV13 and PCV10 contributed to a vaccine-type IPD decrease of $95.4 \%$ (95\% CI 67.0-99.4) and 76.9\% (95\% CI $36.0-91.7)$, respectively, when compared to the pre-immunisation period. The occurrence of NVT between the pre- and postimmunisation periods decreased only in children vaccinated with PCV10 (OR 0.52; 95\% CI 0.05-0.93) but did not change in children vaccinated with PCV13 (OR 1.10; 95\% CI 0.39-2.14). Even when considering the IPD acquisition of NVT and NT, no change was found between both periods in those immunised with either PCV10 or PCV13.

Although during the post-immunisation period, incidence of IPD caused by NVT was significantly lower only in those vaccinated with PCV10 than in unvaccinated children (Table 3), the adjusted odds ratio of 1.75 (95\% CI $0.51-5.98)$ confirmed no difference of acquired NVT-invasive pneumococcal diseases between children immunised with PCV10 and PCV13.

Surprisingly, the IPD burden of VT was not different between unvaccinated and PCV10 vaccinated children, i.e. the OR of 0.70 (95\% CI 0.23-2.07).

Although the population of unvaccinated children under 5 years of age was affected with the same overall IPD burden rate in pre- and post-immunisation periods, i.e. the OR of 1.94 (95\%

Table 1. IPD cases of vaccine (VT), non-vaccine types (NVT) and non-typed cases (NT) in immunised and unimmunised children younger than 5 years in 2012-2013

\begin{tabular}{|c|c|c|c|c|c|c|c|c|}
\hline \multirow{3}{*}{$\begin{array}{l}\text { Child age } \\
\text { (year) }\end{array}$} & \multirow{3}{*}{$\begin{array}{l}\text { Overall IPD } \\
\text { cases }\end{array}$} & \multicolumn{6}{|c|}{ Immunised } & \multirow{3}{*}{ Unimmunised } \\
\hline & & \multicolumn{3}{|c|}{ PCV13 } & \multicolumn{3}{|c|}{ PCV10 } & \\
\hline & & VT & NVT & NT & VT & NVT & NT & \\
\hline 0 & 12 & 0 & 0 & 0 & 1 & 1 & 0 & 10 \\
\hline 1 & 6 & 1 & 1 & 0 & 0 & 1 & 1 & 2 \\
\hline 2 & 6 & 0 & 1 & 1 & 0 & 0 & 0 & 4 \\
\hline 3 & 11 & 0 & 2 & 0 & 1 & 0 & 1 & 7 \\
\hline 4 & 6 & 0 & 1 & 0 & 2 & 0 & 0 & 3 \\
\hline Overall & 41 & 1 & 5 & 1 & 4 & 2 & 2 & 26 \\
\hline
\end{tabular}


Table 2. Odds ratios (OR) and percentage IPD decreases of vaccine (VT) and non-vaccine serotypes (NVT), including nontyped cases (NT) between pre- (2007-2008) and post-immunisation (2012-2013) periods in children <5 years

\begin{tabular}{|c|c|c|c|c|c|c|c|c|}
\hline \multirow{3}{*}{ IPD serotypes } & \multicolumn{4}{|c|}{ PCV13 immunised } & \multicolumn{4}{|c|}{ PCV10 immunised } \\
\hline & \multicolumn{2}{|c|}{ IPD cases } & \multirow{2}{*}{ OR } & \multirow{2}{*}{ Decrease (\%) } & \multicolumn{2}{|c|}{ IPD cases } & \multirow{2}{*}{ OR } & \multirow{2}{*}{ Decrease (\%) } \\
\hline & Pre & Post & & & Pre & Post & & \\
\hline Overall & 70 & 7 & $0.27(0.12-0.58)$ & $73.5(42.3-87.8)$ & 70 & 8 & $0.30(0.15-0.63)$ & $69.7(37.0-85.4)$ \\
\hline VT & 58 & 1 & $0.05(0.01-0.33)$ & $95.4(67.0-99.4)$ & 46 & 4 & $0.23(0.08-0.64)$ & $76.9(36.0-91.7)$ \\
\hline NVT & 12 & 5 & $1.10(0.39-3.14)$ & $-10.5(<0-61.1)$ & 24 & 2 & $0.22(0.05-0.93)$ & $77.9(6.5-94.8)$ \\
\hline$N V T+N T$ & 12 & 6 & $1.33(0.50-3.53)$ & $-32.6(<0-50.2)$ & 24 & 4 & $0.44(0.15-1.27)$ & $55.8(<0-84.7)$ \\
\hline
\end{tabular}

Number of children $<5$ years of age in the pre-immunisation period $(2007-2008) \mathrm{N}=1,020,728$ and in the post-immunisation period $\mathrm{N}=770,000$ vaccinated with either PCV10 or PCV13

Table 3. Adjusted odds ratios (aOR) and percentage IPD decreases between immunised (I) and unimmunised (U) children younger than 5 years in 2012-2013 for vaccine types (VT), non-vaccine types (NVT) and non-typed cases (NT) of IPD

\begin{tabular}{|c|c|c|c|c|c|c|c|c|}
\hline \multirow{3}{*}{ IPD serotypes } & \multicolumn{4}{|c|}{ PCV13 } & \multicolumn{4}{|c|}{ PCV10 } \\
\hline & \multicolumn{2}{|c|}{ IPD cases } & \multirow{2}{*}{$\mathrm{aOR}$} & \multirow{2}{*}{ Decrease (\%) } & \multicolumn{2}{|c|}{ IPD cases } & \multirow{2}{*}{ aOR } & \multirow{2}{*}{ Decrease (\%) } \\
\hline & I & $U$ & & & I & U & & \\
\hline Overall & 7 & 26 & $0.23(0.10-0.53)$ & $76.6(47.5-89.6)$ & 8 & 26 & $0.29(0.14-0.62)$ & $70.8(37.7-86.3)$ \\
\hline VT & 1 & 14 & $0.14(0.04-0.50)$ & $86.3(50.0-96.3)$ & 4 & 7 & $0.70(0.23-2.07)$ & $30.4(<0-76.6)$ \\
\hline NVT & 5 & 4 & $1.03(0.34-3.13)$ & $-2.6(<0-66.4)$ & 2 & 11 & $0.18(0.05-0.65)$ & $82.0(35.3-95.0)$ \\
\hline$N V T+N T$ & 6 & 12 & $0.44(0.17-1.10)$ & $56.4(<0-82.8)$ & 4 & 19 & $0.17(0.06-0.47)$ & $83.1(53.1-94.0)$ \\
\hline
\end{tabular}

CI 0.60-1.48), the balance of vaccine and non-vaccine serotypes changed significantly (Fig. 1). It was revealed that IPD occurrence of 10 vaccine serotypes, included in both commercial vaccines, decreased significantly by $61.5 \%(95 \%$ CI $14.7-82.6)$ in unvaccinated children, when comparing pre- and post-immunisation periods. But the lower vaccination rate against three vaccine pneumococcal serotypes contained only in the PCV13 exhibited an insignificant reduction of $38.9 \%(95 \% \mathrm{CI}<0-65.9)$ against all 13 vaccine serotypes among unimmunised children when comparing both periods. Contrary to the VT reduction, children who received no immunisation faced the likelihood of NVT together with unspecified IPD acquisition, representing an increase of IPD occurrence rate up to $153 \%$ (95\% CI 14-464).

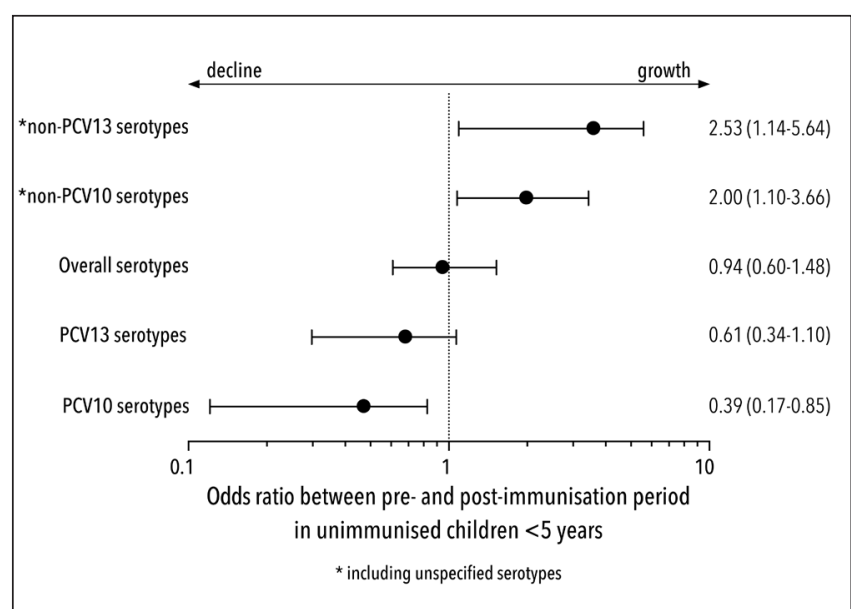

Fig. 1. Odds ratios of vaccine serotypes and non-vaccine including unspecified serotypes of IPD between pre- and postimmunisation periods in unimmunised children.

\section{DISCUSSION}

The routine vaccination against pneumococcal infections conducted since 2010 has changed the epidemiological situation in the Czech Republic, i.e. overall IPD occurrence in children under 5 years of age was reduced by $46.6 \%$ in $2012-2013$. This was the result of vaccination uptake rate reaching $68.6 \%$, in agreement with unpublished data from health insurance companies. Children immunised with PCV13 and PCV10 vaccines were protected against all IPDs with rates of $73.5 \%$ and $69.7 \%$, respectively. These findings are consistent with the results of other recent studies conducted in Brazil, Israel, France, and Norway (7-10).

Interestingly, there was a larger impact of PCV13 than that of PCV10 on the IPD reduction of vaccine serotypes in children under 5 years, documented by an $86 \%$ reduction for PCV13immunised and a $30 \%$ reduction for PCV10-immunised. This could be explained by a shift in IPD distribution of non-PCV10 serotypes in unimmunised children in 2012-2013. There were 7 cases caused by three vaccine serotypes, i.e. 3, 6A and 19A included only in the PCV13 vaccine, and only 4 cases of serotypes not shared by both commercial vaccines.

Conversely, PCV10 immunisation apparently decreased the IPD burden of non-vaccine serotypes while PCV13 did not influence it. However, as no statistically significant difference of non-vaccine serotype acquisition between children immunised with PCV10 and PCV13 was found, it can be assumed that immunisation with both vaccines did not change the occurrence of non-vaccine serotypes in immunised children. The incidence of invasive pneumococcal diseases in the Czech Republic in 2012-2013 did not show a raised IPD occurrence of non-vaccine serotypes in immunised children compared to years 2007-2008. Nevertheless, continued surveillance will be important in order to 
recognise eventual replacement of serotypes, which has already been observed in countries after the introduction of the 7- or 13-valent pneumococcal conjugate vaccine into national routine immunisation schedules (11-13).

Although routine immunisation did not affect IPD burden in unvaccinated children, there was a shift in the IPD occurrence caused by vaccine and non-vaccine serotypes. It was shown that the IPDs of ten serotypes common to both vaccines significantly declined among unvaccinated children by up to $61.5 \%$. Unfortunately, this positive impact of routine immunisation was compensated for by the IPD burden on both non-vaccine and unspecified serotypes, which was nearly 2.5 times higher in the population of unvaccinated children between the pre- and postimmunisation periods. Therefore, unvaccinated children could be considered as a source of pneumococci, especially those of non-vaccine serotypes.

The limitations of these analyses include the lack of knowledge concerning full and partial immunisation as well as concerning a number of received vaccine doses.

Even though routine immunisation has evidently brought about a positive impact on IPD occurrence in the child population, the use of less valent vaccines could reduce this favourable effect. If only one vaccine had been applied, which currently represents the broadest serotype coverage, the results of the immunisation programme would have been even better. Naturally, increasing the vaccination rate is another important means of reducing the reservoir of pneumococci in the child population.

\section{REFERENCES}

1. Motlová J, Beneš Č, Křížová P. Invasive pneumococcal disease in the Czech Republic in 2000-2008. Zpr Epidemiol Mikrobiol. 2009 Jun;18(6):203-9. (In Czech.)

2. Motlová J, Beneš Č, Křížová P. Invasive pneumococcal disease in the Czech Republic in 2009. Zpr Epidemiol Mikrobiol. 2010 Mar;19(3):6877. (In Czech.)

3. Motlová J, Kozáková J, Křížová P. Invasive pneumococcal disease in the Czech Republic in 2010. Zpr Cent Epidemiol Mikrobiol. 2011 Feb;20(2):64-9. (In Czech.)
4. Motlová J, Beneš Č, Kozáková J, Křŕžová P. Invasive pneumococcal disease in the Czech Republic in 2011. Zpr Cent Epidemiol Mikrobiol. 2012 Feb;21(2):51-8. (In Czech.)

5. Kozáková J, Motlová J, Beneš Č, Šebestová H, Křížová P. Invasive pneumococcal disease in the Czech Republic in 2012. Zpr Cent Epidemiol Mikrobiol. 2013 Mar;22(3):97-104. (In Czech.)

6. Kozáková J, Šebestová H, Křížová P. Invasive pneumococcal disease in the Czech Republic in 2013. Zpr Cent Epidemiol Mikrobiol. 2014 Mar;23(3):89-97. (In Czech.)

7. Domingues CM, Verani JR, Montenegro Renoiner EI, de Cunto Brandileone MC, Flannery B, de Oliveira LH, et al.; Brazilian Pneumococcal Conjugate Vaccine Effectiveness Study Group. Effectiveness of tenvalent pneumococcal conjugate vaccine against invasive pneumococcal disease in Brazil: a matched case-control study. Lancet Respir Med. 2014 Jun;2(6):464-71.

8. Ben-Shimol S, Greenberg D, Givon-Lavi N, Schlesinger Y, Somekh E, Aviner S, et al. Early impact of sequential introduction of 7-valent and 13 -valent pneumococcal conjugate vaccine on IPD in Israeli children $<5$ years: an active prospective nationwide surveillance. Vaccine. 2014 Jun 5;32(27):3452-9.

9. Levy C, Varon E, Picard C, Béchet S, Martinot A, Bonacorsi S, et al. Trends of pneumococcal meningitis in children after introduction of the 13-valent pneumococcal conjugate vaccine in France. Pediatr Infect Dis J. 2014 Dec;33(12):1216-21.

10. Steens A, Bergsaker MA, Aaberge IS, Rønning K, Vestrheim DF. Prompt effect of replacing the 7-valent pneumococcal conjugate vaccine with the 13 -valent vaccine on the epidemiology of invasive pneumococcal disease in Norway. Vaccine. 2013 Dec 16;31(52):6232-8.

11. Waight PA, Andrews NJ, Ladhani SN, Sheppard CL, Slack MP, Miller E. Effect of the 13-valent pneumococcal conjugate vaccine on invasive pneumococcal disease in England and Wales 4 years after its introduction: an observational cohort study. Lancet Infect Dis. 2015 May;15(5):535-43.

12. Choi YH, Jit M, Gay N, Andrews N, Waight PA, Melegaro A, et al. 7-Valent pneumococcal conjugate vaccination in England and Wales: is it still beneficial despite high levels of serotype replacement? PLoS One. 2011;6(10):e26190. doi: 10.1371/journal.pone.0026190.

13. Miller E, Andrews NJ, Waight PA, Slack MP, George RC. Herd immunity and serotype replacement 4 years after seven-valent pneumococcal conjugate vaccination in England and Wales: an observational cohort study. Lancet Infect Dis. 2011 Oct;11(10):760-8.

Received November 4, 2014 Accepted in revised form March 24, 2016 